\title{
Autonomía de la voluntad y estado de derecho: libertad o sometimiento administrativo ante la ley
}

Autonomy of the will and rule of law: freedom or administrative submission before the law

1 José Luis Vásconez Fuentes Universidad Técnica de Ambato

jl.vasconez@uta.edu.ec

Artículo de Investigación Científica y Tecnológica

Enviado: 11/12/2021

Revisado: $26 / 12 / 2021$

Aceptado: 28/01/2022

Publicado:05/02/2022

DOI: https://doi.org/10.33262/ap.v4i1.1.158

Cítese:

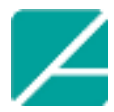

Ciencia Digital

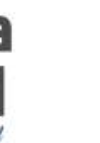

Vásconez Fuentes, J. L. (2022). Autonomía de la voluntad y estado de derecho: libertad o sometimiento administrativo ante la ley . AlfaPublicaciones, 4(1.1), 267278. https://doi.org/10.33262/ap.v4i1.1.158

ALFA PUBLICACIONES, es una Revista Multidisciplinar, Trimestral, que se publicará en soporte electrónico tiene como misión contribuir a la formación de profesionales competentes con visión humanística y crítica que sean capaces de exponer sus resultados investigativos y científicos en la misma medida que se promueva mediante su intervención cambios positivos en la sociedad. https://alfapublicaciones.com

La revista es editada por la Editorial Ciencia Digital (Editorial de prestigio registrada en la Cámara Ecuatoriana de Libro con No de Afiliación 663) www.celibro.org.ec

Esta revista está protegida bajo una licencia Creative Commons Attribution Non Commercial No

Derivatives 4.0 International. Copia de la licencia: http://creativecommons.org/licenses/by-nc-nd/4.0/ 


\section{Palabras claves:} democracia, autonomía, libertad, estado de derecho

\section{Keywords:}

democracy, autonomy, freedom, rule of law

\section{Resumen}

Latinoamérica ha vivido diversos episodios jurídicos sociales que han servido de precedente para que sus sociedades se forjen de acuerdo al marco institucional que cada país ha impuesto, y que en la mayoría de los casos reconocen al pueblo como su máximo exponente con poder soberano, digno de libertad y democracia. El objetivo general de esta investigación es analizar si la autonomía de la voluntad y el estado de derecho es parte de la libertad o un sometimiento administrativo ante la ley, la metodología utilizada es de diseño bibliográfico y de tipo documental. La autonomía de la voluntad y estado de derecho, son aspectos relevantes de la política y la sociedad, donde se ha visto olvidada, a pesar de que constitucionalmente se pretende afirmar que son derechos propios de un pueblo democrático. Sin embargo, sus conceptos han cambiado con el paso del tiempo, a conveniencia e intereses individuales Como conclusión se menciona que la autonomía de la voluntad jurídica en la nueva normalidad y antes, ya se la reconocía como un pequeño rango de acción jurídico general, que gira en torno al pensamiento y la libre elección del individuo como actor social. En este sentido el ciudadano, debe adaptarse al orden legal de derecho local que se le otorgue, y esto dependerá en gran medida del tipo de mandatario que tenga el país. Por tanto, se puede expresar que la autonomía de la voluntad y el estado de derecho a pesar de ser muestras de democracia y libertad siempre estarán sometidas a norma y leyes rigurosas a los que los pueblos siempre estarán sometidos, producto del poder que tiene la ley establecida en cada uno de los países donde conviven. 
type. The autonomy of the will and the rule of law are relevant aspects of politics and society, where it has been forgotten, despite the fact that constitutionally it is intended to affirm that they are the rights of a democratic people. However, its concepts have changed over time, at convenience and individual interests. In conclusion, it is mentioned that the autonomy of the legal will in the new normality and before, was already recognized as a small range of general legal action, which revolves around the thought and the free choice of the individual as a social actor. In this sense, the citizen must adapt to the legal order of local law that is granted to him, and this will depend largely on the type of president that the country has. Therefore, it can be expressed that the autonomy of the will and the rule of law, despite being signs of democracy and freedom, will always be subject to rigorous norms and laws to which the people will always be subject, as a result of the power that the established law has. in each of the countries where they live.

\section{Introducción}

Latinoamérica ha vivido diversos episodios jurídicos sociales que han servido de precedente para que sus sociedades se forjen de acuerdo al marco institucional que cada país ha impuesto, y que en la mayoría de los casos reconocen al pueblo como su máximo exponente con poder soberano, digno de libertad y democracia.

Sin embargo, en ocasiones al momento de que el pueblo desea ejercer sus derechos, a veces se ve truncado este derecho de autonomía, y voluntad por parte de las instituciones legales que en ocasiones se ven violadas las garantías constitucionales.

La libertad como menciona Castro-Montero (2017), es entendida desde dos perspectivas la negativa y la positiva, en el caso de la primera es entendida como la falta de autoridad donde no hay obstáculos para ejecutar cualquier acción, desde la perspectiva positiva, es se refiere a la capacidad que tiene un individuo de poder decir sobre su propio futuro. En este sentido expresarse libremente es uno de los elementos primordiales en una democracia.

A nivel político, la libertad de expresión permite que los ciudadanos expresen sus pensamientos acerca de las figuras públicas, para hacer ver la existencia de democracia y libertad de poder expresar la voluntad del pueblo, es por ello que se hace necesario 
mantener siempre este vinculo entre sociedad y gobierno. Sin embargo, esto no es del todo cierto puesto que la libertad ha sido siempre condicionada de acuerdo a los estándares y necesidades desde el punto de vista político y deja de ser una libertad absoluta y universal (Castro-Montero, 2017).

Es así como Cardoso (2017), menciona que la democracia es:

La expresión de la voluntad de los pueblos a través de sus gobernantes. Esto solo ocurre en sociedades en las que impera el Estado de Derecho, es decir, el sometimiento a una legalidad constituida; por lo tanto, no se puede hablar de ella en un Estado dictatorial. (pág. 299)

La democracia es un modelo donde existe libertad y exigencias, para mantener un equilibrio justo y saludable en cada uno de los poderes del gobierno sin el abuso en alguno de ellos, sin caer en tantas complejidades, o en mucha simplicidad pues ambos extremos son causales de rebelión social o de una política dominante (Innerarity, 2020).

De esta manera, Munck (2016), Saffon \& Urbinati (2013 citado por Monsivais-Carrillo, 2020) menciona que:

El régimen democrático puede adoptar distintas configuraciones institucionales, pero no puede ser menos que un sistema de reglas y procedimientos que haga valer los derechos y libertades de la ciudadanía. Como mínimo, debe constituir un sistema competitivo de elecciones periódicas y regulares, el acceso al poder público y el ejercicio de la autoridad estatal, que tenga respaldo en un régimen de libertades y derechos efectivos.

Ecuador es un país que ha pasado por diversos episodios jurídicos sociales con marcados aspectos que también le permitieron a su sociedad conformarse rigiéndose por normativas establecidas en su Constitución, reconociendo un Estado de democracia y libertad.

De esta manera el objetivo de esta investigación es analizar si la autonomía de la voluntad y el estado de derecho es parte de la libertad o un sometimiento administrativo ante la ley, la metodología utilizada es de diseño bibliográfico y de tipo documental.

\section{Metodología}

Esta investigación está basada en un diseño bibliográfico donde se aplican procesos lógicos para analizar, resumir a través de un proceso de abstracción científica, realizando una recopilación de información que permitan el desarrollo de la investigación, realizando este proceso de forma ordenada con objetivos precisos (Palella \& Martins, 2010)

Asimismo, la investigación es de tipo documental pues se enfoca de forma exclusiva en seleccionar información de diferentes fuentes bibliográficas para obtener datos precisos 
y necesarios a través de procesos de búsqueda que puedan garantizar confiabilidad en la información recabada.

\section{Resultados y Discusión}

\section{Autonomía de la Voluntad}

Para Hinostroza (2014) citado por Ortega (2020), la autonomía etimológicamente hablando es "poder darse a si mismo normas", que van de la mano con el negocio jurídico donde los particulares se "les concibe autonomía como poder reconocido"

La palabra autonomía según Torres (2012) deriva del griego "autos" que significa hacer algo por si mismo, y del griego "nomos" cuyo significado es ley. Por tanto, de forma doctrinaria la autonomía es como autonomía de la voluntad y autonomía privada, regida por la normativa establecida por la escuela francesa, y en cuyo caso muchos países de Latinoamérica se rigen por ella, tal es el caso de Ecuador.

Desde un punto de vista filosófico la autonomía representa la modernidad, cuyo período le da importancia al hombre como el centro del conocimiento, dotado de razón y el poder de decidir y actuar según sus valores morales (San Vicente, 2016). El hombre en su afán de buscar su camino y lograr su progreso recurre a herramientas e instrumentos que apoyen sus decisiones.

La voluntad según Torres (2012) existe desde el origen del hombre, y desde aquellos tiempos ha sido generadora de conflictos y desacuerdos producto del logro de intereses propios antes que los de la sociedad, hasta que con el pasar del tiempo y la creación del Derecho esto fue decayendo y se lograron establecer leyes y doctrinas que han ayudado a dilucidar la concepción de la voluntad de los individuos, como parte de la libertad de elección, la ética y moral.

Para Kant la autonomía de la voluntad expresada en su obra titulada "Fundamentación de la Metafísica de las Costumbres", indica que la metafísica de las costumbres debe indagar sobre la idea de que la voluntad del hombre sea pura y posible San Vicente (2016), y que vaya mas allá de solo los sentimientos o emociones del hombre.

En este sentido Kant manifiesta que siendo racional es como el hombre tiene el poder de actuar conforme a las leyes, basadas en principios provenientes de la voluntad para tomar decisiones, por cuanto es necesaria la razón que permita crear una voluntad buena (San Vicente, 2016).

De esta manera se aprecia que la autonomía de la voluntad requiere de gran responsabilidad, de tener sentido de culpa para obrar correctamente y de creer que también el hombre es capaz de hacer lo contrario. Y es que, a través de la autonomía de la voluntad es que los particulares a través de sus relaciones jurídicas gestionan sus 
intereses privados rigiéndose por las normativas del Estado, y este a su vez les hace cumplir sus reglas (Córdoba, 2021)

La autonomía de la voluntad es la libertad que tienen las partes de disponer legal y constitucionalmente para realizar contratos o acuerdos que permitan crear o modificar sus propias relaciones jurídicas (Núñez, 2018). Asimismo, es importante resaltar que existen restricciones dentro del derecho contractual al momento de realizar estos acuerdos entre las partes y que deben ser considerados para evitar el incumplimiento de las normas establecidas por los entes correspondientes.

La autonomía y la libertad tienen estrecha relación y según la Corte Interamericana de Derechos Humanos menciona lo citado por (Conde, 2017):

"El concepto de libertad y la posibilidad de todo ser humano de auto determinarse y escoger libremente las opciones y circunstancias que le dan sentido a su existencia, conforme a sus propias opciones y convicciones, es un derecho humano básico, propio de los atributos de la persona, que se proyecta en toda la Convención Americana"

Considerando que la libertad es un derecho fundamental de cada ser humano, y que se establece como tal en la mayoría de las constituciones del mundo, que como principal antecedente de la libertad estaba afianzado en las Institutas de Justiniano donde se menciona que: "Libertad, de la que viene la denominación de libres, es la natural facultad de cada cual para hacer lo que le plazca, a no ser que por la fuerza o por la ley se le prohíba" (Torres, 2012). Sin embargo, con el pasar del tiempo esto se ha ido adaptando pasando por la libertad del derecho humano, y luego por la adoptada por la revolución francesa, hasta el concepto que se tiene hoy en día y que se rige según las leyes del Estado y la sociedad"

\section{Estado de derecho}

Para las Naciones Unidas (2022) según el secretario general, el estado de derecho lo define como:

Un principio de gobernanza en el que todas las personas, instituciones y entidades, públicas y privadas, incluido el propio Estado, están sometidas a leyes que se promulgan públicamente, se hacen cumplir por igual y se aplican con independencia, además de ser compatibles con las normas y los principios internacionales de derechos humanos. Asimismo, exige que se adopten medidas para garantizar el respeto de los principios de primacía de la ley, igualdad ante la ley, separación de poderes, participación en la adopción de decisiones, legalidad, no arbitrariedad, y transparencia procesal y legal. 
De hecho, este concepto está incluido en la Carta de las Naciones Unidas afirmando que los objetivos de esta organización es la de tener condiciones adecuadas para lograr mantener la justicia, respecto y las obligaciones derivadas de todos los tratados y acuerdos del derecho internacional (Naciones Unidas, 2022).

El Estado de derecho, está ligado a un sistema de gobierno donde exista democracia, y que es legítima a través de las decisiones que toman las autoridades del Estado, por tanto, aquellas leyes establecidas desde un gobierno democrático, también significa que los ciudadanos deben cumplirlas y que son obligatorias (Pineda \& Merelo, 2020).

Según la revista electrónica gestión de Multiplica Ediciones (2021), el país esta en el lugar 92 de 139 países que han sido evaluados en referencia al índice de Estado de derecho que realiza la Organización World Justice Project (WJP). El Estado de derecho es aquel donde las leyes son fundamentales, sin embargo, el país no ha tenido índices elevados durante muchos años, producto de los controles inadecuados en referencia al crimen y el conflicto civil.

En Ecuador existe un Estado plurinacional según lo menciona el artículo N. ${ }^{\circ} 1$ de la carta magna, donde se expresa que debe existir un Estado de derecho, un país soberano, democrático y con justicia social, con un tipo de gobierno descentralizado y conformado como república (Pazmiño, 2019). En concordancia con lo anteriormente expuesto, la población ecuatoriana debe acatar y cumplir lo estipulado en el artículo N. ${ }^{\circ} 1$, por tanto, es importante que cada una de las partes respalden el Estado plurinacional con el propósito de mantener la República.

En este sentido las principales características que deben existir en un Estado de derecho según Oroz (2015) son:

- División de poderes: en el Estado de Derecho el ejercicio del poder se divide entre los diversos organismos de poder público. Estableciéndose entre ellos una relación de coordinación y cooperación.

- Control y fiscalización de los poderes públicos: en el Estado de Derecho los ciudadanos están facultados para vigilar y supervisar la labor de las entidades públicas. Del mismo modo, las instituciones tienen el deber de controlarse mutuamente (Legislativo-Ejecutivo-Judicial).

- Imperio de la ley: en el estado de Derecho ningún hombre se encuentra por encima de lo que dispone la ley. En otras palabras, en el Estado de Derecho no existen reyes o reinas cuya voluntad se impone sobre lo que la ley ordena. Ello es así porque la ley es expresión directa de la voluntad del pueblo soberano, producto de la participación de los ciudadanos y sus representantes. 
- Derechos y libertades fundamentales: en el Estado de Derecho se reconocen positivamente, garantizan y protegen los derechos humanos velando por que estos puedan ser ejercidos a cabalidad por sus titulares.

Al inmiscuirse analíticamente es imposible evitar hablar desde un punto de vista filosófico-jurídico como piedra angular para descifrar el verdadero trasfondo de la libre voluntad y el acatamiento obligatorio del sistema de derecho, como instrumento regulatorio y generativo de la sociedad institucionalizado en las últimas generaciones.

\section{Democracia ante la autonomía de la voluntad}

El concepto de democracia fue tomado formalmente en Grecia por misóginos, que le dieron a este tipo de sistema características racistas, sexistas y excluyentes (Esteva, 2020), donde solo un pequeño número de ciudadanos eran los encargados de tomar las decisiones, y las mujeres, las personas con cualquier discapacidad y los esclavos no tenían derecho a expresarse libremente.

Al llegar la democracia a Estados Unidos está también mantenía esta línea sexista, y que permitió la construcción de este régimen al resto del mundo, hasta que finalmente con el pasar del tiempo y muchos procesos gubernamentales donde se aceptó un estado de nación moderno y se adopto al pensamiento de la revolución francesa.

Si bien es cierto que la democracia es un sistema donde el estado le da valor e importancia al pueblo, esto es un tanto contradictorio, pues las decisiones son tomadas por el gobierno, y en todo caso cuando los pueblos ejercen sus decisiones en cuando deben realizar su derecho al voto.

En este sentido la soberanía no es uniforme pues a pesar de crear leyes para el pueblo no existe igualdad ni uniformidad en las decisiones, ideologías y creencias truncando en gran medida el Estado de derecho. Por lo tanto, el término Democracia, debe ser nuevamente analizado y ajustado dentro del verdadero Estado de derecho, autonomía de la voluntad e imperio de la Ley, donde se nota la existencia de una monarquía maquillada que evita el ejercicio de la plena libertad y albedrio de las personas en el mundo entero, donde aun se cometen barbaries, delitos y las leyes no se cumplen adecuadamente.

\section{Autonomía y voluntad nuevo paradigma en derecho administrativo}

Actualmente la sociedad ha enfrentado diversos cambios producto de la "nueva normalidad" por tanto la autonomía de la voluntad deberá adecuarse a estas nuevas situaciones, enfrentarse a nuevos retos dentro del marco jurídico, cumpliendo con las obligaciones de manera que la sociedad vaya adaptándose a nuevas doctrinas que les permita sobrellevar las situaciones actuales. 
Por tanto, se podría afirmar que la autonomía de voluntad descansa sobre la noción de libertad, y que supone un principio de igualdad jurídica para todos, pero que al ser practicada no es igual debido a la falta de igualdad entre los individuos por lo que surge un sometimiento a leyes que no son beneficiosas para todas las partes implicadas.

El único escenario en que la autonomía de la voluntad pueda ser citada sin una doble moral filosófica y jurídica, seria en el cual el término "Poder" no le sea arrebatado a su único y verdadero titular: "El pueblo". Mediante el paradigma de Administración Democrática, que no es más que elegir un esclavizador, con las esperanzas de que este "Mandatario" sea justo y ecuánime, pero indudablemente su mera presencia para imponer su voluntad ineludiblemente se traduce un atropello a la autonomía de la voluntad y al derecho natural del hombre libre e independiente.

\section{Conclusiones}

- La autonomía de la voluntad jurídica en la nueva normalidad y antes, ya se la reconocía como un pequeño rango de acción jurídico general, que gira en torno al pensamiento y la libre elección del individuo como actor social. Esta autonomía administrada por los gobiernos, a través de las leyes, hace que el ciudadano contemporáneo pierda su voluntad autonómica para vivir en sociedad constitucional, rodeada de una prisión de normas, que no lo hace completamente libre, ni independiente, al contrario, solo ser obediente para poderle garantizar "encajar" en la sociedad y no ser señalado por incumplir alguna de las normas o leyes establecidas.

- En este sentido el ciudadano, debe adaptarse al orden legal de derecho local que se le otorgue, y esto dependerá en gran medida del tipo de mandatario que tenga el país, si en caso dado cae en manos de un mandatario progresista, tendrá un cierto mayor espacio de movimiento dentro de los barrotes jurídico sociales que lo rigen, en caso de un gobierno neoliberal estos límites serian menores, pero independientemente de cualquiera de los dos casos posibles de "democracia" los individuos vivirán dentro de un cerco normativo.

- Por tanto, se puede expresar que la autonomía de la voluntad y el estado de derecho a pesar de ser muestras de democracia y libertad siempre estarán sometidas a norma y leyes rigurosas a los que el pueblo siempre estará sometido, producto del poder que tiene la ley establecida en cada uno de los países donde conviven. 


\section{Referencias Bibliográficas}

Cardoso, J. (2017). Democracia y redes sociales. Universidad-Verdad, (72), 297-311.

Castro-Montero, J. (2017). Libertad de expresión y límites democráticos. Revista de Derecho $6,11-25$.

Conde, J. (2017). La legitima hereditaria, su contradicción con la autonomía de la voluntad. Trabajo especial de grado de la Universidad Empresarial Siglo 21.

Córdoba, Y. (2021). El plazo de la liquidación del contrato estatal: tensión entre competencia y autonomía de la voluntad. Bogotá, Colombia: Trabajo especial de grado de la Universidad San Tomás para optar al títrulo de maestría en Derecho Contractual Público y Privado.

Esteva, G. (2020). Protegiendo la autonomía de la democracia. Utopía y Praxis Latinoamericana 25(91) , 252-264.

Innerarity, D. (2020). Una teoría de la democracia compleja. Gobernar en el siglo XXI. Barcelona, España: Galaxia Gutenberg.

Monsivais-Carrillo, A. (2020). La indiferencia hacia la democracia en América Latina. ÍCONOS Revista de Ciencias Sociales 66(24) , 151-171.

Multiplica Ediciones. (29 de Octubre de 2021). ¿Es el Ecuador un Estado de derecho?

Naciones Unidas. (2022). ¿Qué es el Estado de Derecho?

Núñez, M. (2018). Las limitaciones a la autonomía de la voluntad. Guayaquil, Ecuador: Trabajo especial de grado de la Universidad Católica de Santiago de Guayaquil para optar al título de Abogado de los tribunales y Juzgados de la República del Ecuador.

Oroz, E. (17 de Agosto de 2015). ¿Qué es Estado de Derecho y cuales son sus características?

Ortega, D. (2020). La autonomía de la voluntad como derecho constituciona y clausulas abusivas como parte de la asimetría informativa del consumidor en el perú. Huaraz, Ancash, Perú: Trabajo especial de grado de la Universidad Nacional "Santiago Antunez de Mayolo" para optar al título de Doctor en Derecho y Ciencias Políticas.

Palella, S., \& Martins, F. (2010). Metodología de la Investigación Cuantitativa. Carcas, Venezuela: FEDUPEL, Fondo Editorial de la Universidad Pedagógica Experimental Libertador. 
Pazmiño, M., Noboa, G., \& Yánez, T. (2019). La ética decolonial y su relación con el Estado de derecho en cuanto a la interculturalidad y la plurinacionalidad en el Ecuador. Uisrael Revista Científica 6(3), 55-70.

Pineda, I., \& Merelo, V. (15 de Abril de 2020). Estado de derecho o estado de naturaleza. Recuperado el 23 de Enero de 2022, de La seguridad jurídica en tiempos de emergencia: https://derechoecuador.com/estado-de-derecho-o-estado-denaturaleza/

San Vicente, A. (2016). El principio de autonomía de la voluntad. Informe de la asignatura Teoría del Derecho de la UNAM.

Torres, J. (2012). Quebrantamiento del principio de la autonomía de la voluntad dentro de los contratos de adhesión el Ecuador. Quito, Ecuador: Trabajo especial de grado de la Universidad Internacional SEK para optar al título de Abogado.

\section{LCiencia}


El artículo que se publica es de exclusiva responsabilidad de los autores y no necesariamente reflejan el pensamiento de la Revista Alfa Publicaciones.

\section{Ciencia}

El artículo queda en propiedad de la revista y, por tanto, su publicación parcial y/o total en otro medio tiene que ser autorizado por el director de la Revista Alfa Publicaciones.
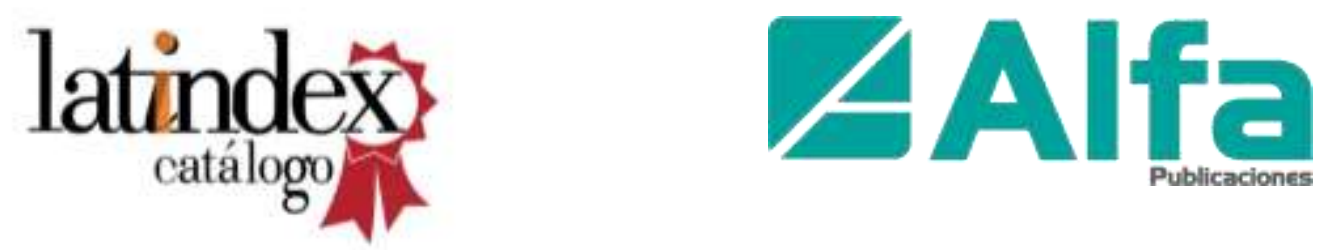

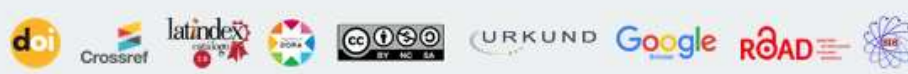
DLatinREV

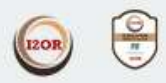

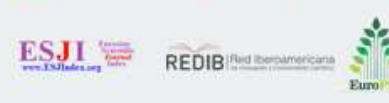

$\underbrace{}_{\text {wizdom.ai }} \mathrm{O}_{\text {OpenAIRE }}^{+}$

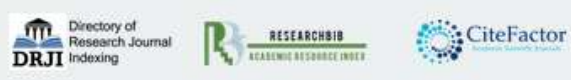

